\title{
Reformulating time-dependent density functional theory with non-orthogonal localized molecular orbitals $\dagger$
}

\author{
Ganglong Cui, ${ }^{a b}$ Weihai Fang ${ }^{b}$ and Weitao Yang*a \\ Received 12th August 2009, Accepted 19th October 2009 \\ First published as an Advance Article on the web 13th November 2009 \\ DOI: $10.1039 / \mathrm{b} 916688 b$
}

\begin{abstract}
Time-dependent density functional theory (TDDFT) has broad application in the study of electronic response, excitation and transport. To extend such application to large and complex systems, we develop a reformulation of TDDFT equations in terms of non-orthogonal localized molecular orbitals (NOLMOs). NOLMO is the most localized representation of electronic degrees of freedom and has been used in ground state calculations. In atomic orbital (AO) representation, the sparsity of NOLMO is transferred to the coefficient matrix of molecular orbitals (MOs). Its novel use in TDDFT here leads to a very simple form of time propagation equations which can be solved with linear-scaling effort. We have tested the method for several long-chain saturated and conjugated molecular systems within the self-consistent charge density-functional tight-binding method (SCC-DFTB) and demonstrated its accuracy. This opens up pathways for TDDFT applications to large bio- and nano- systems.
\end{abstract}

\section{Introduction}

Time-dependent density functional theory (TDDFT) has been widely applied to the calculations of electronic excitations, response properties, and transport in molecules and bulk, because it is an effective approach. ${ }^{2,5,15,17,19-21}$ While the computational cost of TDDFT is moderate in contrast to the wave-function-based approaches, it still scales as $O\left(N^{4}\right)$ $\left[O\left(N^{3}\right)\right.$ with the density fitting technique] where $N$ is the number of molecular orbitals. ${ }^{25}$ A better scaling approach is needed for applications to large biological and nano systems where there are many frontiers to explore.

Indeed, within the ground state DFT, ${ }^{11,18}$ the first linearscaling approach was developed by Yang based on a divideand-conquer (DAC) strategy. ${ }^{29,31}$ While the DAC method is efficient and continues to be used and further improved, ${ }^{13,34}$ there have been many new developments of linear scaling methods based on minimization with respect to the density matrix, ${ }^{9}$ orthogonal localized molecular orbitals (OLMO) ${ }^{23}$ and nonorthogonal localized molecular orbitals NOLMO. ${ }^{30}$ Within TDDFT, a linear-scaling TDDFT algorithm named the localized density matrix (LDM) had been developed by Chen and coworkers; ${ }^{33}$ it employed the "nearsighted" property of the first-order response density matrix in atomic orbital (AO) representation and was implemented by various semi-empirical methods, as well as first-principles TDDFT. ${ }^{26}$ Compared with linear response TDDFT calculations which are only valid in linear reponse regime, ${ }^{3}$ the time-domain TDDFT can be more useful, suitable for not only linear

${ }^{a}$ Department of Chemistry, Duke University, Durham,

NC 27708-0346, USA. E-mail: weitao.yang@duke.edu;

Tel: (919)660-1562; Fax: (919)660-1605

${ }^{b}$ Chemistry College, Beijing Normal University, Beijing 100875,

China

$\dagger$ Electronic supplementary information (ESI) available: Experimental details; AO data. See DOI: 10.1039/b916688b response areas but also strong laser field, etc. Its extensive applications are the best examples. $4,6,27,28,32$

We here focus on NOLMO, which is the most localized representation of electron because the localized molecular orbitals, which are linear independent, need not be orthogonal. The absence of orthogonality constraints allows the molecular orbitals to be the most localized ones. For molecular systems, the NOLMOs have been demonstrated to be better localized than the corresponding OLMOs with a spatial spread reduced by $10-28 \%,{ }^{8}$ and this advantage has also been realized in the recent ground-state linear scaling calculations. ${ }^{1}$ In this work, we reformulate the TDDFT in terms of nonorthogonal localized molecular orbitals. As NOLMO is the most localized representation of electronic degrees of freedom, it can be most efficient in computation and is particularly useful for linear scaling calculations. In addition, our method, based on timepropagation, can describe all time dependences for application beyond the linear response regime.

\section{NOLMO-TDDFT equations}

\section{A Formulation}

In this section, we reformulate original TDDFT equations in terms of NOLMOs. Within the conventional TDDFT method, ${ }^{21}$ the time-dependent evolution of the interacting electron density can be obtained as the solution to the set of single-particle time-dependent Kohn-Sham equations:

$$
i \hbar \frac{\partial\left|\phi_{i}\right\rangle}{\partial t}=H_{\mathrm{s}}\left|\phi_{i}\right\rangle,
$$

in which the time-dependent electron density is expressed as $\rho(r, t)=\sum_{i}^{\text {occ }}\left|\phi_{i}(r, t)\right|^{2}$, the Kohn-Sham Hamiltonian is given by $\hat{H}_{\mathrm{s}}={ }^{i}-\frac{1}{2} \nabla^{2}+V_{\text {ext }}(r, t)+\int \frac{\rho\left(r^{\prime}, t\right)}{\left|r-r^{\prime}\right|} d r^{\prime}+V_{\mathrm{xc}}(r, t)$ where $-\frac{1}{2} \nabla^{2}$, $\int \frac{\rho\left(\mathbf{r}^{\prime}, t\right)}{\left|\mathbf{r}-\mathbf{r}^{\prime}\right|} d \mathbf{r}^{\prime}, V_{\text {ext }}$ and $V_{\mathrm{xc}}$ denote the kinetic energy, Coulombic, 
external and exchange-correlation potentials, respectively. The molecular orbital $\phi_{i}$ should be orthogonal such that $\left\langle\phi_{i} \mid \phi_{j}\right\rangle=\delta_{i j}$. Based on this set of equations, several approaches have been developed, including the Casida eigenvalue matrix method, ${ }^{10}$ the Sternheimer approach, ${ }^{24}$ and the real-space methods in time domain. ${ }^{4,5,17}$

Alternatively, the evolution of KS electron density operator with respect to time, in Heisenberg picture, can be expressed as

$$
i \hbar \frac{d \hat{\rho}(t)}{d t}=\left[H_{\mathrm{s}}, \hat{\rho}(t)\right]
$$

which is independent of the specific representation, including CMO and NOLMO. Furthermore, this equation of motion for density operator $\rho(t)$ is equivalent to eqn (1), under the following conditions

$$
\hat{\rho} \hat{\rho}=\hat{\rho}
$$

and

$$
\operatorname{Tr} \hat{\rho}=N
$$

which ensures that $\hat{\rho}$ represents a Slater determinant.

With the orthogonal MO (OMO), $\left\{\left|\phi_{i}\right\rangle\right\}$, the density operator can be expressed as the following

$$
\hat{\rho}(t)=\sum_{i=1}^{N}\left|\phi_{i}\right\rangle\left\langle\phi_{i}\right| .
$$

Normally, for the initial density matrix, one uses the canonical molecular orbitals (CMOs) that are the eigenstates of the ground state Kohn-Sham Hamiltonian. However, orthogonal localized molecular orbitals (OLMOs) can also be used because both CMOs and OLMOs share the same expression of density operator, eqn (3), as a result of MO orthogonality, $\left\langle\phi_{i} \mid \phi_{j}\right\rangle=\delta_{i j}$. Note that the usual TDDFT equations, eqn (1), can also be derived from eqn (2) with density operator expression, eqn (3).

Density operator, $\rho(t)$, can also be constructed with NOLMOs, $\left\{\left|\psi_{i}\right\rangle\right\},{ }^{30}$ which are the most localized representation of electronic degrees of freedom, because the orthogonality constraints between MOs are removed among NOLMOs. With NOLMO, the density operator is expressed as ${ }^{30}$

$$
\hat{\rho}(t)=\sum_{i, j=1}^{N}\left|\psi_{i}\right\rangle S_{i j}^{-1}\left\langle\psi_{j}\right|
$$

where $S_{i j}{ }^{-1}$ is the $i j$ element of the inverse of overlap matrix $\mathbf{S}$ of NOLMO with $S_{i j}=\left\langle\psi_{i} \mid \psi_{j}\right\rangle$. The density operator expressed with NOLMOs, eqn (4), is consistent with the wave function of a Slater determinant because it satisfies three conditions: normalization $\operatorname{Tr}=N$ ( $N$ is total number of electron), idempotency $\hat{\rho}^{2}=\hat{\rho}$ and Hermitian $\hat{\rho}=\hat{\rho}^{\dagger}$. ${ }^{30}$

We now reformulate the usual TDDFT equations, eqn (1), in terms of NOLMOs. Using the derivation of eqn (4) with respect to time, we obtain

$$
\frac{\partial \hat{\rho}}{\partial t}=\sum_{i, j=1}^{N}\left\{\frac{\partial\left|\psi_{i}\right\rangle}{\partial t} S_{i j}^{-1} \psi_{j}+\left|\psi_{i}\right\rangle S_{i j}^{-1} \frac{\partial\left\langle\psi_{j}\right|}{\partial t}+\left|\psi_{i}\right\rangle \frac{\partial S_{i j}^{-1}}{\partial t}\left\langle\psi_{j}\right|\right\} .
$$

With the relation, $\mathbf{S S}^{-1}=\mathbf{I}$, and its derivation with respect to time, $\frac{\partial \mathbf{S}^{-1}}{\partial t}=-\mathbf{S}^{-1} \frac{\partial \mathbf{S}}{\partial t} \mathbf{S}^{-1}$, we have

$$
\begin{aligned}
\frac{\partial S_{i j}^{-1}}{\partial t} & =-S_{i k}^{-1} \frac{\partial S_{k l}}{\partial t} S_{l j}^{-1} \\
& =-S_{i k}^{-1}\left(\left\langle\frac{\partial\left\langle\psi_{k}\right|}{\partial t} \mid \psi_{l}\right\rangle+\left\langle\psi_{k} \mid \frac{\partial\left|\psi_{l}\right\rangle}{\partial t}\right\rangle\right) S_{l j}^{-1} .
\end{aligned}
$$

After inserting eqn (7) into eqn (5) and employing eqn (2), we arrive at

$$
i \hbar \frac{\partial \hat{\rho}}{\partial t}=i \hbar\left((1-\hat{\rho}) \sum_{i j} \frac{\partial\left|\psi_{i}\right\rangle}{\partial t} S_{i j}^{-1}\left\langle\psi_{j}\right|\right.
$$

$$
\left.+\sum_{i j}\left|\psi_{i}\right\rangle S_{i j}^{-1} \frac{\partial\left\langle\psi_{j}\right|}{\partial t}(1-\hat{\rho})\right)
$$

$$
=\hat{H}_{\mathrm{s}} \hat{\rho}-\hat{\rho} \hat{H}_{\mathrm{s}}
$$

Note that because $\left|\psi_{i}\right\rangle$ is the occupied MO, we can get the following relations

$$
\begin{gathered}
\hat{\rho}\left|\psi_{i}\right\rangle=\left|\psi_{i}\right\rangle \\
(1-\hat{\rho})\left|\psi_{i}\right\rangle=0 \\
\hat{\rho}(1-\hat{\rho})=0 .
\end{gathered}
$$

Then, multiplying $(1-\hat{\rho})$ and $\hat{\rho}$ on eqn (10) from LHS, respectively, and using the relations above, we have

$$
i \hbar(1-\hat{\rho}) \sum_{i j} \frac{\partial\left|\psi_{i}\right\rangle}{\partial t} S_{i j}^{-1}\left\langle\psi_{j}\right|=(1-\hat{\rho}) H_{\mathrm{s}} \hat{\rho}
$$

and

$$
i \hbar \sum_{i j}\left|\psi_{i}\right\rangle S_{i j}^{-1} \frac{\partial\left\langle\psi_{j}\right|}{\partial t}(1-\hat{\rho})=-\hat{\rho} H_{\mathrm{s}}(1-\hat{\rho}),
$$

from which we can see that eqn (14) is the complex conjugate of eqn (15); thus, one equation is enough to completely determine the time-dependent propagation of systems. This means that the time-dependence of NOLMOs contributes the density matrix only through $(1-\hat{\rho}) \frac{\partial\left|\psi_{i}\right\rangle}{\partial t}$, irrelevant to $\hat{\rho} \frac{\partial\left|\psi_{i}\right\rangle}{\partial t}$. Therefore, eqn (14) can become

$$
i \hbar \sum_{i j} \frac{\partial\left|\psi_{i}\right\rangle}{\partial t} S_{i j}^{-1}\left\langle\psi_{j}\right|=H_{\mathrm{s}} \hat{\rho} .
$$

After multiplying $\left|\psi_{k}\right\rangle$ on eqn (16) from RHS, we get our final NOLMO-TDDFT equation

$$
i \hbar \frac{\partial\left|\psi_{k}\right\rangle}{\partial t}=H_{\mathrm{s}}\left|\psi_{k}\right\rangle .
$$

\section{B NOLMO versus CMO}

It is a pleasant surprise that the NOLMO-TDDFT equations, eqn (17), have exactly the same form as the convential TDDFT equations, eqn (1), except for the differences in construction of the electron density or density operator. In 
$\mathrm{CMO} / \mathrm{OLMO}$ the density operator is constructed by eqn (3); however, in NOLMO it is expressed as eqn (4). Though the density operator in NOLMO is more complicated than that in CMO, NOLMOs can lead to linear scaling calculations, because of the optimal localization of MOs. The localization of MOs can lead to different sparse representations in different implementations. In atomic orbital (AO) representation, each $\mathrm{CMO}$ is expanded by a full set of AOs in system, so the coefficient matrix of MOs is also dense; however, as NOLMOs are localized in space, we do not need to have the entire set of AOs to expand each NOLMO. Normally, a small set of AOs is enough for each NOLMO, Fig. 3; thus, the NOLMO sparsity is transferred to the coefficient matrix of MOs, so the coefficient matrix is sparse. In a real-space grid representation, ${ }^{4,17,27}$ the sparsity can be directly employed. Specifically, each NOLMO only uses a small set of grid points in real-space, unlike the situation of $\mathrm{CMO}$ in which each CMO uses all grid points in space.

\section{Linear scaling calculation of $\mathrm{S}^{-1}$}

The computational cost of $\mathbf{S}^{-1}$ in eqn (4) can be reduced to linear scaling by a variational principle $\operatorname{Tr}\left(\mathbf{B S}^{-1}\right)=$ $\min _{X} \operatorname{Tr}[\mathbf{B}(2 \mathbf{X}-\mathbf{X S X})]$ under the condition that $\mathbf{X}=\mathbf{X}^{\dagger}$ with any negative definite matrix $\mathbf{B}$. The minimizer is $\mathbf{X}_{0}=\mathbf{S}^{-1} \cdot{ }^{30}$ For simplicity, we can choose $\mathbf{B}=-\mathbf{S}$ in this work. Alternatively, other approaches of calculating $\mathbf{S}^{-1}$ with linear scaling efforts also exist, taking advantage of the fact that the overlap matrix $\mathbf{S}$ of NOLMOs is sparse. ${ }^{12,16,22}$

\section{On-the-fly relocalization}

Another key point of our algorithm is the "on-the-fly" re-localization technique. This technique is used to maintain the sparsity of NOLMOs during the time propagation, because along time propagation, NOLMOs will become more delocalized and their sparsity will decrease. Before they reach beyond the pre-defined localization region, a re-localization operation is performed on the NOLMO so that they are kept in the localized region. Specifically, in AO representation, our "on-the-fly" relocalization will convert the delocalized, and hence denser MO coefficient matrix into a sparse one, maintaining the sparsity of coefficient matrix during the propagation without loss of accuracy. The extreme localization of NOLMO can be seen in Fig. 2. If no relocalization is done, the sparsity of NOLMOs will be lost in the propagation process and the effort of computation will increase. Therefore, re-localization is necessary in maintaining sparsity, needed for efficiency and linear scaling calculation. Because of the parallelization characteristics of NOLMO's construction, ${ }^{8}$ its performance does not significantly an increase in the computational effort of our algorithm.

\section{Implementation}

In the present work, we choose atomic orbitals to expand NOLMOs, thus the NOLMO sparsity is reflected in the coefficient matrix of MOs. The NOLMO in AO representation can be expressed as

$$
\psi_{i}(t)=\sum_{\mu}^{A O} c_{\mu i}(t) \chi_{\mu}
$$

in which $c_{\mu i}(t)$ is the $i$ th time-dependent NOLMO coefficient, while $\chi_{\mu}$ is the $\mu$ th time-independent AO. After some mathematical transformations when eqn (18) is inserted into eqn (17), the final equation of motion in the $\mathrm{AO}$ representation is

$$
i \hbar \frac{d}{d t} \mathbf{C}=\mathbf{S}^{-1} \mathbf{H C}
$$

where $\mathbf{C}, \mathbf{H}$, and $\mathbf{S}^{-1}$ are AO-based matrices representing the NOLMO coefficient, Hamiltonian and inverse of overlap matrix, respectively. Note that coefficient matrix $\mathbf{C}$ is explicitly time-dependent while $\mathbf{H}$ is a matrix which has time-dependence both explicitly on the time-dependent external field and implicitly on the time-dependent density matrix expressed with NOLMO as shown in eqn (4). While different electronic structure methods have different constructions of the Hamiltonian matrix $\mathbf{H}$ in eqn (19), their time propagation remains same (eqn (19)). In the present work, to demonstrate our NOLMO-TDDFT approach, we implemented it based on the SCC-DFTB approximation to DFT $^{7}$ with a focus on molecular optical excitations.

As a first implementation to test the accuracy of local representation of NOLMO, we simply use the direct inverse for evaluating $\mathbf{S}^{-1}$. Thus our current implementation is not yet linear scaling.

\section{A Truncation of coefficient matrix}

Here we discuss how to localize the MO coefficient matrix in our scheme. Note that although both CMO- and NOLMO-TDDFT equations, eqn (1) and eqn (17), can result into the same equation of motion in $\mathrm{AO}$ representation, eqn (19), the nature of coefficient matrix $\mathbf{C}$ is very different between $\mathrm{CMO} / \mathrm{OLMO}$ and NOLMO. In the CMO picture, the MO coefficient matrix $\mathbf{C}$ is fully delocalized as shown in Fig. 1 because each CMO uses all AOs in system, whereas in NOLMO, it is extremely localized, Fig. 2, because each NOLMO uses a small subset of AOs as shown in Fig. 3. Specifically, each CMO will occupy the whole AO space which are used to expand $\mathrm{MO}$ (LCAO). In contrast, the AO space for each NOLMO is significantly reduced because each NOLMO is extremely localized so that we only need to expand each NOLMO in AOs within a predefined radius. The radius is chosen as a critical parameter for testing the convergence of truncation on MO coefficient matrix $\mathbf{C}$. The centers of these predefined region are the centroids of NOLMOs. In the present work, all NOLMOs use the same radius for the localized regions, and the accuracy of the method will increase with increasing radius. Thus, the localization radius will be a crucial parameter for controlling the accuracy of the calculations. This local expansion of NOLMO is fundamental to achieving linear scaling. Furthermore, although we use long-chain molecules as examples to test the feasibility of our method in this work, we do not foresee any difficulty for extension to 2D and 3D systems. For instance, we can use all AOs located in a predefined sphere region (3D) to expand the corresponding NOLMO. 


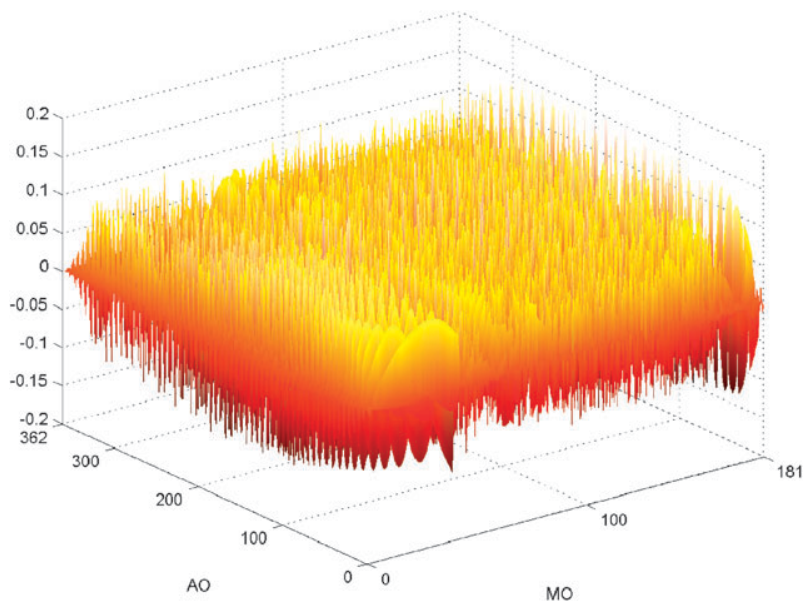

Fig. 1 The real part of CMO distribution in coefficient matrix at the initial time is shown and the imaginary part of it has as similar distribution; the ordinate is the MO coefficient. The molecule is $C_{60} H_{122}$.

\section{B $\delta$-Perturbation on MOs and time propagation scheme}

An initial perturbation method should be chosen to study the time-dependence of the system. In this work, the delta function perturbation technique is employed. This technique has been successfully applied to time-domain TDDFT methods in the past decade. ${ }^{27}$ The perturbing potential of it is $V(r, t)=-k_{0} x_{\mathrm{v}} \delta(t)$, which has the contribution only initially by perturbing the wave-function of NOLMOs: $\psi_{i}\left(r, 0^{+}\right)=\exp \left(i k_{0} x_{\mathrm{v}}\right) \psi_{i}(r)$, where $k_{0}$ is the momentum transferred to the system at the initial time, and a small constant ensuring that the response of system is linear and only dipole transitions are excited, while $x_{\mathrm{v}}$ is the $x$ component of the 3D Cartesian coordinate. The optical excitation energies can be achieved through Fourier transform of the timedependent dipole moments, which are calculated during the propagation. This technique has been extensively employed to extract absorption spectroscopy in time-domain methods. ${ }^{4,6,27}$

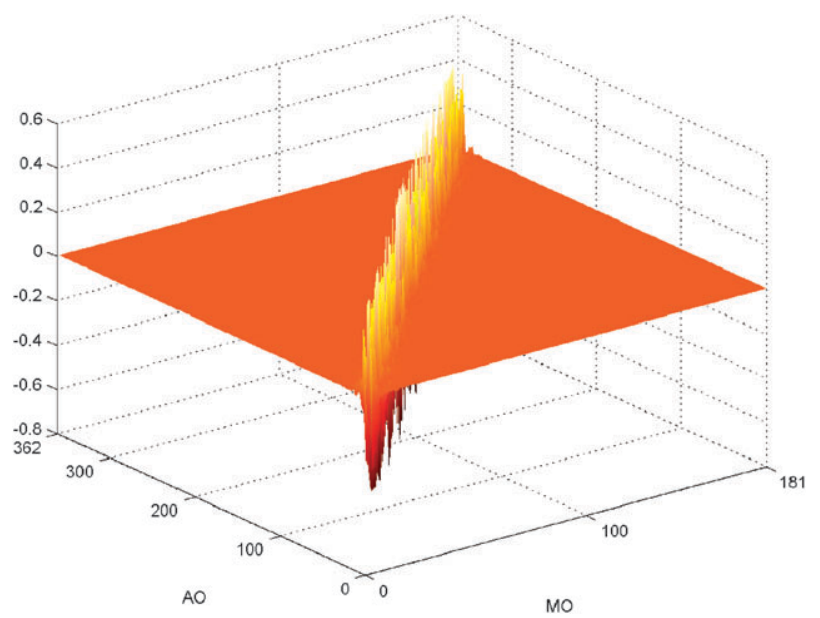

Fig. 2 The real part of NOLMO distribution in coefficient matrix at the initial time is shown and the imaginary part of it has the similar distribution; the ordinate is the MO coefficient. The molecule is $\mathrm{C}_{60} \mathrm{H}_{122}$.

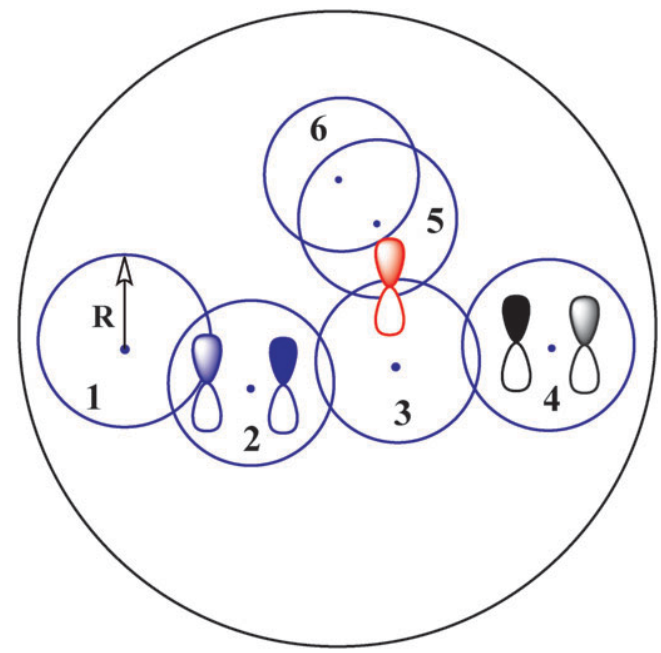

Fig. 3 The big circle represents the total AOs of system; each small circle represents a subspace set of AOs used to expand each NOLMO whose MO centroid is the center of circle; thus, the radius $(R)$ of circle is a critical parameters for implementing linear scaling calculations. 1 , 2, 3, 4, 5 and 6 are the indexes of NOLMOs in systems. In some cases, one AO belongs to two or more NOLMOs (red AO). Note that in the CMO picture, each MO needs all AOs in systems (the big circle), whereas in NOLMO, each MO only requires a small set of AOs controlled by its centroid and radius.

It is an important and complicated problem to choose a proper propagation method in time-domain TDDFT. ${ }^{5}$ Here we focus on demonstrating the feasibility and accuracy of our method, and use the 4-order Runge-Kutta method to propagate the equation of motion.

\section{Algorithm summary}

We summarize our NOLMO-TDDFT's algorithm as follows: (1) initial NOLMOs are calculated using NOLMO construction methods based on previous methods; ${ }^{8,14}$ (2) a set of initially perturbed NOLMOs is generated through the delta perturbation technique; (3) NOLMOs are propagated with respect to time according to eqn (19); (4) if one NOLMO spreads out of a predefined radius as described above, an "on the fly" re-localization of NOLMOs is conducted; ${ }^{8}$ if not, do nothing; (5) repeat the steps from (3) to (5) until the total propagation time is reached.

\section{Results and discussion}

The long chain saturated molecule, $\mathrm{C}_{60} \mathrm{H}_{122}$, and conjugated molecule, $\mathrm{C}_{60} \mathrm{H}_{62}$, are used as examples in this work. At SCC-DFTB level, $\mathrm{C}_{60} \mathrm{H}_{122}$ has 362 AOs and 181 MOs while $\mathrm{C}_{60} \mathrm{H}_{62}$ has 302 AOs and 151 MOs. The initial perturbation strength $k_{0}$ is 0.01 au along the $x$ direction (the long chain direction of molecules). The integration step of the 4-order Runge-Kutta scheme is $0.01 \mathrm{au}$. The total propagation time is: 100 au with $\mathrm{C}_{60} \mathrm{H}_{122}$ and 200 au with $\mathrm{C}_{60} \mathrm{H}_{62}$. A series of radius values are employed to truncate the coefficient matrix $\mathbf{C}$ in eqn (19). The interval time of re-localization is $1 \mathrm{au}$.

Compared with CMO and OLMO, the advantage of NOLMO is its extreme localization. ${ }^{8}$ Fig. 2 represents the NOLMOs' distribution in coefficient matrix space of $\mathrm{C}_{60} \mathrm{H}_{122}$. 


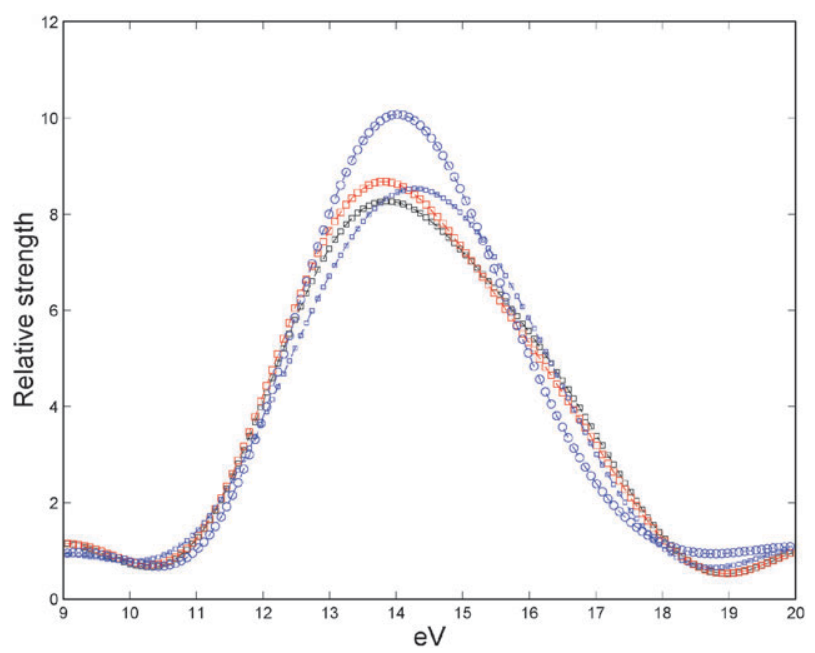

Fig. 4 The main peak of absorption spectroscopy of $\mathrm{C}_{60} \mathrm{H}_{122}$ with different radius values. Black diamonds: no cutoff; red diamonds: $20 \mathrm{au}$; blue circles: $16 \mathrm{au}$; blue diamonds: $14 \mathrm{au}$.

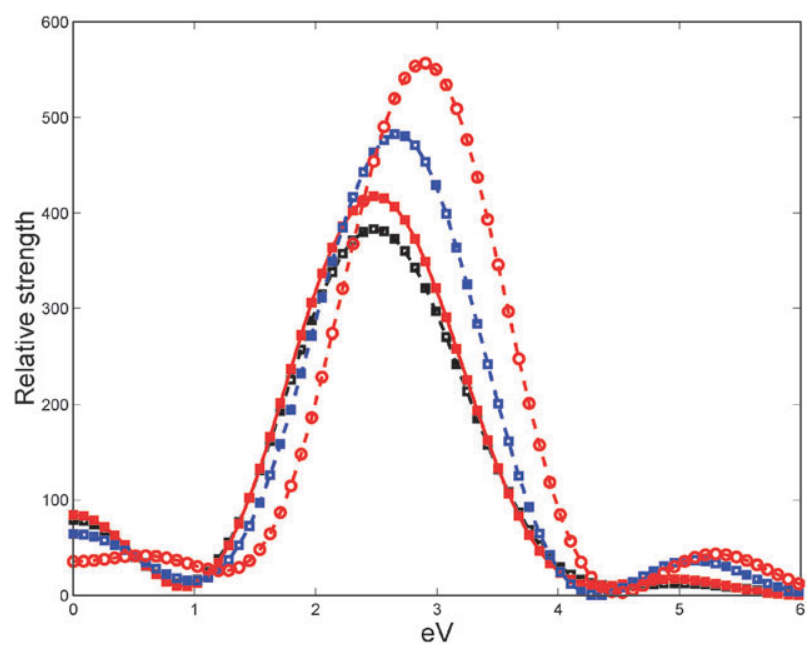

Fig. 5 The main peak of absorption spectroscopy of $\mathrm{C}_{60} \mathrm{H}_{62}$ with different radius values. Black diamonds: no cutoff (or 32, 34 and $36 \mathrm{au}$ ); red diamonds: $30 \mathrm{au}$; blue diamonds: $26 \mathrm{au}$; red circles: $22 \mathrm{au}$.

Table 1 The absorption energies of main peak with different radius values in this work. The corresponding absorption energies calculated from B3LYP/6-31G (BLYP/6-31G) in frequency domain are 2.81 (2.52) eV in $\mathrm{C}_{60} \mathrm{H}_{62}$. The absorption energy of $\mathrm{C}_{60} \mathrm{H}_{122}$ in $\mathrm{LDM}$ (time-domain TDDFT) of Chen et al. is about $13.5 \mathrm{eV}^{28}$

\begin{tabular}{llll}
\hline Radius/au & $\mathrm{C}_{60} \mathrm{H}_{122} / \mathrm{eV}$ & Radius/au & $\mathrm{C}_{60} \mathrm{H}_{62} / \mathrm{eV}$ \\
\hline 12 & 13.80 & 22 & 2.91 \\
16 & 13.90 & 26 & 2.65 \\
20 & 13.85 & 30 & 2.48 \\
22 & 13.85 & 32 & 2.48 \\
No cutoff & 13.85 & No cutoff & 2.48 \\
\hline
\end{tabular}

Because CMO distribution covers the full coefficient matrix space, Fig. 1, there is no sparsity which can be employed for linear scaling calculations. In contrast, the distribution of NOLMOs is much sparser than that of CMOs. This sparsity of NOLMOs can be employed to significantly reduce the computational effort. This sparse characteristic of NOLMOs is not limited to the saturated molecules but is also shared by the conjugated systems. Further details can be found in the ESI. $\dagger$

We check the convergence behavior of our NOLMOTDDFT algorithm using different radius values which define the localization regions of NOLMOs, Table 1. For $\mathrm{C}_{60} \mathrm{H}_{122}$ and $\mathrm{C}_{60} \mathrm{H}_{62}$, the calculated absorption spectroscopies of the main peak are shown in Fig. 4 and Fig. 5, respectively. It is clear that the error of NOLMO-TDDFT decreases with the increase of the radius values as shown in Table 1. Compared with the saturated molecule, a larger radius value is required for conjugated molecules because of strong delocalization of $\pi$ bonds. Based on these results, we can conclude that our NOLMO-TDDFT algorithm can achieve accurate results if a proper radius value is chosen.

\section{Conclusion}

We have reformulated TDDFT equation based on NOLMO, such that it can be used in time-domain calculations with linear scaling efforts. The NOLMO-TDDFT equation, eqn (17), has the same form as the conventional TDDFT equation, eqn (1), except for the different density operator constructions, eqn (3) and eqn (4). With the "on the fly" relocalization technique, the sparsity of NOLMOs is maintained during the propagation process. We have not developed the linear scaling implementation in this current work, but we focus on testing the accuracy of our reformulated TDDFT equation based on NOLMO. We demonstrated the feasibility of our algorithm with the long chain saturated and conjugated systems using the SCC-DFTB method: the accuracy of our method can be controlled by increasing the radius of localization for NOLMO and very good results have been obtained with small radius. Extension to other electronic structure methods and propagation methods is possible. Our development presented here should contribute to TDDFT studies of large-scale bio- and nano-systems. Assistance from Dr Xiangqian $\mathrm{Hu}$ is appreciated. Support from the National Scholarship Foundation of Chinese Ministry of Education (GC) and from the National Science Foundation (WY) is gratefully acknowledged.

\section{References}

1 S. K. Burger and W. T. Yang, Linear-scaling quantum calculations using non-orthogonal localized molecular orbitals, J. Phys.: Condens. Matter, 2008, 20, 294209.

2 M. E. Casida, Recent Advances in Density Functional Methods, World Scientific, Singapore, 1995.

3 M. E. Casida, C. Jamorski, K. C. Casida and D. R. Salahub, Molecular excitation energies to high-lying bound states from time-dependent density-functional response theory: Characterization and correction of the time-dependent local density approximation ionization threshold, J. Chem. Phys., 1998, 108(11), 4439-4449.

4 A. Castro, H. Appel, M. Oliveira, C. A. Rozzi, X. Andrade, F. Lorenzen, M. A. L. Marques, E. K. U. Gross and A. Rubio, Octopus: a tool for the application of time-dependent density functional theory, Phys. Status Solidi B, 2006, 243(11), 2465-2488.

5 A. Castro, M. A. L. Marques and A. Rubio, Propagators for the time-dependent Kohn-Sham equations, J. Chem. Phys., 2004, 121, $3425-3433$. 
6 G. H. Chen, S. Yokojima, W. Z. Liang and X. J. Wang, Localizeddensity-matrix method and its application to nanomaterials, Pure Appl. Chem., 2000, 72(1-2), 281-291.

7 M. Elstner, T. Frauenheim, E. Kaxiras, G. Seifert and S. Suhai, A self-consistent charge density-functional based tight-binding scheme for large biomolecules, Phys. Status Solidi B, 2000, 217(1), 357-376.

8 Huasheng Feng, Jiang Bian, Lemin Li and W. T. Yang, An efficient algorithm for nonorthogonal localized molecular orbitals, J. Chem. Phys., 2004, 120, 9458-9466.

9 E. Hernandez, M. J. Gillan and C. M. Goringe, Linear-scaling density-functional-theory technique, The density-matrix approach, Phys. Rev. B: Condens. Matter, 1996, 53(11), 7147-7157.

10 C. Jamorski, M. E. Casida and D. R. Salahub, Dynamic polarizabilities and excitation spectra from a molecular implementation of time-dependent density-functional response theory: N-2 as a case study, J. Chem. Phys., 1996, 104(13), 5134-5147.

11 W. Kohn and L. J. Sham, Self-consistent equations including exchange and correlation effects, Phys. Rev., 1965, 140, A1133.

12 F. R. Krajewski and M. Parrinello, Stochastic linear scaling for metals and nonmetals, Phys. Rev. B: Condens. Matter Mater. Phys., 2005, 71, 233105.

13 Tai-Sung Lee, Darrin York and W. T. Yang, Linear-scaling semiempirical quantum calculations for macromolecules, J. Chem. Phys., 1996, 105, 2744-2750.

14 Shubin Liu, Jose M. Perez-Jorda and W. T. Yang, Nonothorgonal localized molecular orbitals in electronic structure theory, J. Chem. Phys., 2000, 112, 1634-1644.

15 C. A. Marques, M. A. L. Ullrich, F. Nogueira, A. Rubio, K. Burke and E. K. U. Gross, Time-Dependent Density Functional Theory, Springer, 2006

16 A. M. N. Niklasson, Iterative refinement method for the approximate factorization of a matrix inverse, Phys. Rev. B, Nov. 2004, 70(19), 4.

17 G. Onida, L. Reining and A. Rubio, Electronic excitations: density-functional versus many-body Green's-function approaches, Rev. Mod. Phys., 2002, 74, 601-659.

18 R. G. Parr and W. Yang, Density-Functional Theory of Atoms and Molecules, Oxford University Press, New York, 1989.

19 M. J. G. Peach, C. R. Le Sueur, K. Ruud, M. Guillaume and D. J. Tozer, TDDFT diagnostic testing and functional assessment for triazene chromophores, Phys. Chem. Chem. Phys., 2009, 11, 4465-4470.

20 M. Petersilka, U. J. Gossmann and E. K. U. Gross, Excitation energies from time-dependent density-functional theory, Phys. Rev. Lett., 1996, 76, 1212.
21 E. Runge and E. K. U. Gross, Density-functional theory for timedependent systems, Phys. Rev. Lett., 1984, 52(12), 997-1000.

22 S. Schweizer, J. Kussmann, B. Doser and C. Ochsenfeld, Linearscaling Cholesky decomposition, J. Comput. Chem., 2008, 29, 1004.

23 C. K. Skylaris, A. A. Mostofi, P. D. Haynes, O. Dieguez and M. C. Payne, Nonorthogonal generalized Wannier function pseudopotential plane-wave method, Phys. Rev. B: Condens. Matter Mater. Phys., 2002, 66(3), 12.

24 R. M. Sternheimer, Electronic polarizabilities of ions from the Hartree-Fock wave functions, Phys. Rev., 1954, 96(4), 951-968.

25 S. J. A. Van Gisbergen, C. F. Guerra and E. J. Baerends, Towards excitation energies and (hyper)polarizability calculations of large molecules. Application of parallelization and linear scaling techniques to time-dependent density functional response theory, J. Comput. Chem., 2000, 21, 1511.

26 F. Wang, C. Y. Yam, G. H. Chen and K. N. Fan, Density matrix based time-dependent density functional theory and the solution of its linear response in real time domain, J. Chem. Phys., Apr 2007, 126(13), 7

27 K. Yabana, T. Nakatsukasa, J. I. Iwata and G. F. Bertsch, Realtime, real-space implementation of the linear response time-dependent density-functional theory, Phys. Status Solidi B, 2006, 243(5), 1121-1138.

28 C. Y. Yam, S. Yokojima and G. H. Chen, Linear-scaling timedependent density-functional theory, Phys. Rev. B, Oct 2003, 68(15), 4.

29 W. T. Yang, Direct calculation of electron density in densityfunctional theory, Phys. Rev. Lett., 1991, 66, 1438-1441.

$30 \mathrm{~W}$. T. Yang, Absolute energy minimum principles for linearscaling electronic structure calculations, Phys. Rev. B: Condens. Matter, 1997, 56, 9294-9297.

$31 \mathrm{~W}$. T. Yang and Tai-Sung Lee, A density-matrix divide-andconquer approach for electronic structure calculations of large molecules, J. Chem. Phys., 1995, 103, 5674-5678.

32 S. Yokojima and G. H. Chen, Linear scaling calculation of excitedstate properties of polyacetylene, Phys. Rev. B: Condens. Matter Mater. Phys., 1999, 59(11), 7259-7262.

33 S. Yokojima and G. H. Chen, Linear-scaling localizeddensity-matrix method for the ground and excited states of onedimensional molecular systems, Chem. Phys. Lett., 1999, 300(5-6), 540-544.

34 Darrin York, Taisung Lee and W. T. Yang, Quantum mechanical treatment of biological macromolecules in solution using linearscaling electronic structure methods, Phys. Rev. Lett., 1998, 80, 5011-5014. 